Archive for

Organic Chemistry

Arkivoc 2018, part iii, 229-239

\title{
Synthesis of new chiral bis-imidazolidin-4-ones: comparison between the classic method and green chemistry conditions
}

\author{
Nadia Bouzayani, ${ }^{a}$ Wassima Talbi, ${ }^{a}$ Sylvain Marque, ${ }^{b *}$ Yakdhane Kacem, ${ }^{a *}$ and Béchir Ben Hassine ${ }^{a}$ \\ ${ }^{a}$ Laboratoire de Synthèse Asymétrique et Catalyse Homogène, Faculté des Sciences, Université de Monastir, \\ Avenue de l'Environnement, 5019 Monastir, Tunisie \\ ${ }^{b}$ Université de Versailles Saint-Quentin-en-Yvelines, Institut Lavoisier de Versailles (ILV), UMR CNRS 8180, \\ 45 avenue des Etats-Unis, 78035 Versailles Cedex, France \\ Email: sylvain.marque@uvsa.fr, yakacem@yahoo.com
}

Received 08-06-2017

Accepted 12-29-2017

Published on line 03-15-2018

\section{Abstract}

Novel bis-imidazolidin-4-ones were synthesized in moderate to good yields through the cyclocondensation of $o-, m$ - and $p$-phthalaldehydes with various substituted phenylhydrazides. These nitrogenated cyclic compounds were prepared via green chemistry conditions using microwave irradiation.

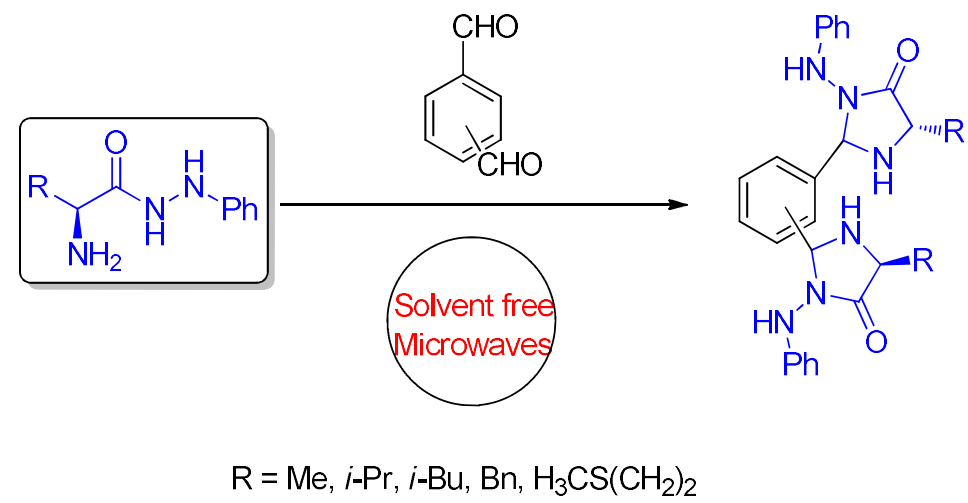

Keywords: Bis-imidazolidin-4-ones, cyclocondensation, phenylhydrazides 


\section{Introduction}

Nitrogen heterocycles are key building blocks for a large number of medicinally-relevant molecules. They constitute an important core fragment in different natural products and pharmaceutical agents. ${ }^{1}$ The chemistry of imidazolidinones has recently attracted more attention due to their reactivity and several biological properties. They have been regarded as anthelmintic, ${ }^{2}$ analgesic, ${ }^{3}$ antibacterial, ${ }^{4}$ antifungal, ${ }^{5}$ antiviral, ${ }^{6}$ antitubercular ${ }^{7}$ and anticancer effects, ${ }^{8}$ and also as a new chemical class of herbicide. ${ }^{9}$ Imidazolidinones can be used as hydrolytically cleavable precursors for the controlled release of fragrant aldehydes and ketones. ${ }^{9}$ Considerable efforts have recently been devoted to the development of more efficient approaches for the preparation of imidazolidinone derivatives. They have generally been obtained by condensation of $\alpha$-aminoamides with carbonyl compounds (aldehyde or ketone). ${ }^{10}$ Among these preparations, several studies concerning the synthesis of chiral imidazolidin-4-ones derivatives containing two stereogenic centers using classical conditions have previously been described in papers by Milos. ${ }^{11-13}$

As part of our ongoing efforts directed toward the synthesis of heterocyclic compounds starting from $\alpha$ amino acids ${ }^{14-19}$ and our studies on the reactivity of $\alpha$-amino_acid phenylhydrazides, ${ }^{20,21}$ we describe herein a practical and efficient synthetic pathway for the preparation of bis-imidazolidin-4-ones using a green chemistry context (solvent free and without catalyst conditions). To the best of our knowledge, no synthesis of bis-imidazolidin-4-ones have been reported with $\alpha$-amino acid phenylhydrazide derivatives as starting materials. ${ }^{1}$ On the other hand, green or sustainable chemistry has become, over time, a research concept for the development of environmentally and eco-friendly chemistry using products, chemical processes and synthetic pathways that decrease the production of hazardous substances. So, microwave irradiation (MW) is one of the potential green chemistry techniques used during the recent years. The main benefits of performing the reaction under microwave conditions are the higher product yields and the significant rateenhancements that can be observed. We have used, in this study, a new microwave synthesis system (startSYNTH) that combines sophisticated design and high technology with ease of use and safety.

\section{Results and Discussion}

The starting L- $\alpha$-amino acid phenylhydrazides 3a-e were prepared in a manner similar to the well-known procedure described by Verardo et al. ${ }^{22}$ The commercially available L- $\alpha$-amino acid ester hydrochlorides 1a-e reacted with phenylhydrazine (2) under mild conditions affording the corresponding phenylhydrazides 3a-e in good yields (Scheme 1, Table 1).

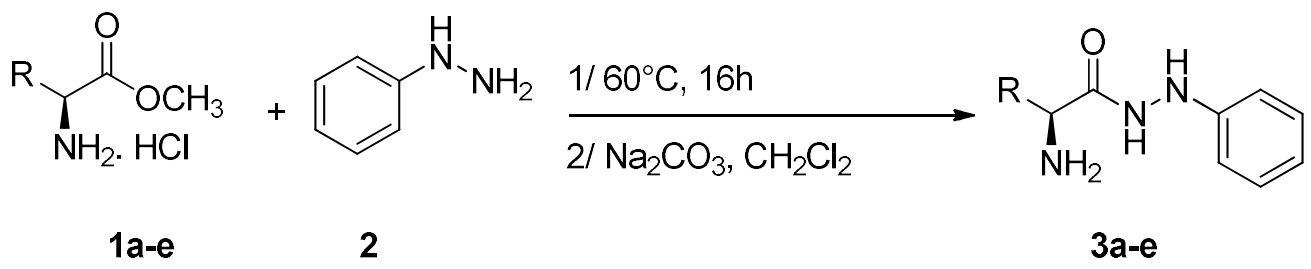

Scheme 1. Synthesis of substituted $\alpha$-amino acid phenylhydrazides 3a-e.

For the investigation of optimal conditions of the coupling reactions, 0 -phthalaldehyde $4 \mathrm{a}$ and L-alanine phenylhydrazide 3a were employed as reactants (Scheme 2). Two procedures were compared using the same reacting mixtures: the conventional heating with a usual acidic catalyst and in a microwave-assisted approach. First, the use of sulfuric acid or acetic acid as catalysts led to low yields (Table 2, entries 1-3). However, $p$ toluenesulfonic acid brought about a double cyclization up to $94 \%$, mainly due to the use of a higher 
temperature, allowing the water vaporization from the appropriate solvent (toluene) and favoring reduced contact of water with the organic materials (entries 4-6). The role of the solvent is tricky to estimate, since it is related to the nature of the catalyst. At first, reactants are not completely soluble in toluene at $70{ }^{\circ} \mathrm{C}$ and no reaction was occurred with PTSA or sulfuric acid (results not depicted in table 2); a temperature of $100{ }^{\circ} \mathrm{C}$ needed to perform reactions in this solvent. Regardless with the solvent, the reactions conducted with PTSA are much cleaner by TLC compared to $\mathrm{H}_{2} \mathrm{SO}_{4}$ where a big spot was observed for this latter on the baseline, added to numerous new spots. In fact, unidentified side products appeared with sulphuric acid and were insoluble in the medium, likely due to opened forms of the condensation products. To further examine the reactivity of the dialdehyde $4 a$, we tried the reaction in the absence of catalyst, but no formation of the desired product was observed (entry 7).

Table 1. Synthesis of $\alpha$-aminoacid phenylhydrazides 3a-e

\begin{tabular}{ccccc}
\hline Entry & Product & $\mathrm{R}$ & {$[\alpha]^{a}$} & ${\text { Yield }(\%)^{b}}^{b}$ \\
\hline 1 & $3 \mathbf{a}$ & $\mathrm{Me}$ & +34.2 & 85 \\
2 & 3b & $i-\mathrm{Pr}$ & +22.8 & 72 \\
3 & $3 \mathbf{c}$ & $i-\mathrm{Bu}$ & +30.8 & 70 \\
4 & 3d & $\mathrm{CH}_{2} \mathrm{Ph}$ & +39.4 & 85 \\
5 & 3e & $\left(\mathrm{CH}_{2}\right)_{2} \mathrm{SMe}$ & +29.8 & 79 \\
\hline
\end{tabular}

${ }^{a}$ Values were measured in $\mathrm{MeOH}(\mathrm{c} 0.2) .{ }^{b}$ Isolated product yield.<smiles>O=Cc1ccccc1C=O</smiles>

$4 a$
$+2$<smiles>CC(N)C(=O)NNc1ccccc1</smiles>

3a<smiles>C[C@@H]1NC(c2ccccc2C2N[C@H](C)C(=O)N2Nc2ccccc2)N(Nc2ccccc2)C1=O</smiles>

$5 a$

Scheme 2. Condensation of $o$-phthalaldehyde $4 \mathbf{a}$ and L-alanine phenylhydrazide $3 \mathbf{a}$.

The generalization of the double condensation was next examined with various $\alpha$-amino acid-derived phenylhydrazides 3a-e reacting with the 3 isomeric dialdehydes $4 a-c$ (Scheme 3 and Table 3). The structure of the resulting bis-imidazolidin-4-ones 5a-0 was undoubtedly confirmed based on their analytical and spectral data. The new compounds 5a-o were isolated as a mixture of two inseparable diastereoisomers (TLC showed always a single spot whatever the eluents used); the duplication of signals on the ${ }^{13} \mathrm{C}-\mathrm{NMR}$ spectrum corroborated the presence of two diastereoisomers. It is worth noting that among the three conceivable diasteroisomers (see supporting information), only two were observed in a 1:1 ratio although the attribution of the stereochemistries of the neo-formed stereocenters was not possible. 
Table 2. Optimization of reaction conditions for the coupling of o-phthalaldehyde $4 \mathrm{a}$ and L-alanine phenylhydrazide $\mathbf{3 a}$

\begin{tabular}{cccccc}
\hline Entry $^{\text {a }}$ & Catalyst $(\mathrm{mol} \%)$ & Temp. $\left.^{\circ} \mathrm{C}\right)$ & Time $(\mathrm{h})$ & Solvent & Yields (\% in 5a) \\
\hline 1 & $\mathrm{AcOH}(1)$ & 70 & 24 & EtOH & Trace \\
2 & $\mathrm{H}_{2} \mathrm{SO}_{4}(1)$ & 70 & 24 & EtOH & $<10$ \\
3 & $\mathrm{H}_{2} \mathrm{SO}_{4}(1)$ & 100 & 24 & Toluene & $<10$ \\
4 & $\mathrm{PTSA}(1)$ & 70 & 10 & EtOH & $<10$ \\
5 & $\mathrm{PTSA}(1)$ & $85-90$ & 24 & Toluene & $\mathrm{NR}^{b}$ \\
6 & $\mathrm{PTSA}(1)$ & 100 & 24 & Toluene & $94^{a}$ \\
7 & - & 100 & 24 & Toluene & $\mathrm{NR}^{b}$ \\
\hline
\end{tabular}

${ }^{a}$ Conditions: L-alanine phenylhydrazide $(2 \mathrm{mmol})$, o-phthalaldehyde $(1 \mathrm{mmol})$ under argon. ${ }^{b}$ No reaction occurred.

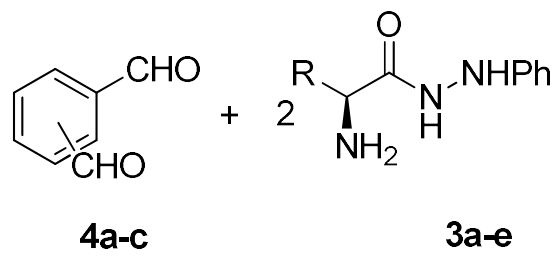

Scheme 3. Synthesis of the bis-imidazolidin-4-ones 5a-o.

In conventional heating, the role of the solvent was crucial; thus, for the reaction performed with the ophthalaldehyde $\mathbf{4 a}$ or the $m$-phthalaldehyde $\mathbf{4 b}$ only a non-polar solvent (toluene) was employed, whereas with the $p$-phthalaldehyde $4 \mathbf{c}$, a mixture of toluene and ethanol was required due to the lesser solubility of paraphthalaldehyde in toluene compare to the ortho isomer. Moreover, among the phenylhydrazide derivatives 3a-e, the experiments conducted with L-valine phenylhydrazide $\mathbf{3 b}$ led to low yields (entries 2,7 and 12), where the two isopropyl moieties on the resulting products may cause steric hindrance, thermodynamically disfavouring the formation of these corresponding bisimidazolidinones $\mathbf{5 b} \mathbf{b} \mathbf{5} \mathbf{g}, \mathbf{5 l}$. To avoid these limitations, the development of an efficient alternative combining higher yields, shorter reaction times and greener conditions (mild, practical, solvent free) was highly desirable. We had originally hoped that the $o^{-}$, $m$ - and $p$-phthalaldehydes could be converted into the corresponding bis-imidazolidin-4-ones with a non classical heating. Moving to a methodology using microwave irradiation at $100{ }^{\circ} \mathrm{C}$ in both solvent- and catalyst free conditions, reactions were completed in only 6 minutes affording the expected bis-imidazolidin-4-ones $\mathbf{5 a - 0}$ in higher yields. We were pleased to find that these conditions efficiently proceeded to afford the desired products without any trace of the starting dialdehydes or the monocondensation product. The heterocycles 5a-o are very silica gel sensitive compounds and numerous attempts failed to isolate them even though basifying the silica gel with triethylamine, in all cases the corresponding starting dialdehydes 4a-c were 
recovered. We succeeded in isolating a mixture of the two diastereoisomers by crystallization using dichloromethane and diethyl ether (7:3). The workup was considerably simpler since a simple crystallization from the crude rapidly gave the targeted heterocycles.

Table 3. Coupling of the phenylhydrazides 3a-e with the dialdehydes 4a-c

\begin{tabular}{cccccc}
\hline Entry & Substrate & Reactant & Product & ${\text { Yields } \Delta(\%)^{a}}$ & Yields MW (\%) $^{b}$ \\
\hline 1 & 4a & 3a & $\mathbf{5 a}$ & 94 & 96 \\
2 & 4a & 3b & $\mathbf{5 b}$ & 34 & 55 \\
3 & 4a & 3c & $\mathbf{5 c}$ & 79 & 81 \\
4 & 4a & 3d & $\mathbf{5 d}$ & 65 & 66 \\
5 & 4a & 3e & $\mathbf{5 e}$ & 60 & 72 \\
6 & 4b & 3a & $\mathbf{5 f}$ & 82 & 93 \\
7 & 4b & 3b & $\mathbf{5 g}$ & 32 & 46 \\
8 & 4b & 3c & $\mathbf{5 h}$ & 67 & 88 \\
9 & 4b & 3d & $\mathbf{5 i}$ & 69 & 71 \\
10 & 4b & 3e & $\mathbf{5 j}$ & 71 & 80 \\
11 & 4c & 3a & $\mathbf{5 k}$ & 59 & 45 \\
12 & 4c & 3b & $\mathbf{5 l}$ & 11 & 32 \\
13 & 4c & 3c & $\mathbf{5 m}$ & 71 & 84 \\
14 & 4c & 3d & $\mathbf{5 n}$ & 78 & 89 \\
15 & 4c & 3e & $\mathbf{5 0}$ & 67 & 80 \\
\hline
\end{tabular}

${ }^{a}$ Conventional heating (oil bath) in toluene $(10 \mathrm{~mL})$ for $o$-phthalaldehyde 4 a or else in toluene/EtOH $(10 \mathrm{~mL})$ for $p$-phthalaldehyde 4 c. ${ }^{b}$ Conditions of activation: microwave irradiation (startSYNTH), $100{ }^{\circ} \mathrm{C}, 6 \mathrm{~min}$.

The conditions under which the reactions summarised in Table 2 were performed are as follows:

Entries 1-5: o-phthalaldehyde 4a $(1.4 \mathrm{mmol}), \alpha$-amino acid phenylhydrazide 3 ( $2.8 \mathrm{mmol})$, PTSA (10 \% mol), $100^{\circ} \mathrm{C}, 24 \mathrm{~h}$ under argon.

Entries 6-10: $m$-phthalaldehyde $4 \mathrm{~b}(1.4 \mathrm{mmol}), \alpha$-amino acid phenylhydrazide $(2.8 \mathrm{mmol})$, PTSA (10 \% mol), $100^{\circ} \mathrm{C}, 24 \mathrm{~h}$ under argon.

Entries 11-15: $p$-phthalaldehyde 4c (1.4 mmol), $\alpha$-amino acid phenylhydrazide 3a-e (2.8 mmol), PTSA (10 \% mol), $100{ }^{\circ} \mathrm{C}, 24 \mathrm{~h}$ under argon.

\section{Conclusions}

In conclusion, we have developed a straightforward and efficient method for the synthesis of new bisimidazolidin-4-ones in moderate to good yields under catalytic conditions. The improvement in processing was pursued up to an approach using the solvent free microwave irradiation in soft conditions (no metal and no catalyst) leading to original nitrogenated compounds with respect to the green chemistry conditions and an easy-to-operate procedure, including the purification by crystallization. 


\section{Experimental Section}

General. All reagents and chemicals were purchased from Sigma-Aldrich chemical company and Acros Organics. Solvents used in reactions were dried and distilled before use. Toluene was distilled over sodium metal, and $\mathrm{EtOH}$ was distilled over $\mathrm{Mg} / \mathrm{I}_{2}$. Nuclear magnetic resonance (NMR) was recorded on Bruker AC-300 spectrometers $\left({ }^{1} \mathrm{H}\right.$ at $300 \mathrm{MHz}$ and ${ }^{13} \mathrm{C}$ at $\left.75 \mathrm{MHz}\right)$ in deuterochloroform $\left(\mathrm{CDCl}_{3}\right)$ as solvent. NMR chemical shifts were calibrated on the solvent residual signal at $7.26 \mathrm{ppm}$ for ${ }^{1} \mathrm{H}$ and at $77.16 \mathrm{ppm}$ for ${ }^{13} \mathrm{C}$. In most cases, the ${ }^{13} \mathrm{C}$ NMR spectra showed a duplication of the chemical shifts signals (presence of two diastereoisomers) and are described herein in this sense, therefore with the twice carbon number vis-à-vis the formula weight. Infrared spectra (IR) were obtained using a Perkin Elmer spectrometer in the range $4000-400 \mathrm{~cm}^{-1}$. Electrospray ionisation (ESI) mass spectroscopy data of compounds $\mathbf{5 a}, \mathbf{5 c}, \mathbf{5 d}, \mathbf{5 e}, \mathbf{5 k}, \mathbf{5 m}, \mathbf{5 n}$, $\mathbf{5 0}$ were recorded on an UPLC Waters device (in positive mode); for the voltages of the mass spectroscopies, the following abbreviations are used: C Capillary (kV), SC Sampling Cone, EC Extraction Cone. Calibration was performed with sodium formate (range from 100 to $1000 \mathrm{~g} \cdot \mathrm{mol}^{-1}$ ) and the lockspray (lockmass on the leucine encephaline $556.2771 \mathrm{~g} . \mathrm{mol}^{-1}$ ) was used without collision energy; the relative intensity of peaks is given in brackets. Electrospray ionisation (ESI) mass spectroscopy data of compounds $\mathbf{5} \mathbf{b}, \mathbf{5} \mathbf{f}, \mathbf{5} \mathbf{h}, \mathbf{5 i}$ and $\mathbf{5} \mathbf{j}$ were recorded on a $Q$ exactive hybrid quadrupole-orbitrap mass spectrometer coupled to a U3000 LC device; the spray voltage was $3000 \mathrm{~V}$. The positive ion calibration was performed with a commercially available mixture of caffeine, MRFA, Ultramark1621 and n-butylamine in a acetonitrile /methanol/acetic acid solution. Characterisation of the compounds 5a-o described herein is for thermal experiment; yields for conventional heating and microwave activation are given in Table 3.

\section{General procedure for the synthesis of bis-imidazolidin-4-ones 5}

Catalytic conditions. To a stirred solution of the $\alpha$-amino acid phenylhydrazide 3a-e $(2.8 \mathrm{mmol}, 2 \mathrm{eq})$ and the $o$-phthalaldehyde $4 \mathbf{a}, \mathrm{m}$-phthalaldehyde $\mathbf{4 b}$ or the $p$-phthalaldehyde $4 \mathrm{c}(187.7 \mathrm{mg}, 1.4 \mathrm{mmol}, 1 \mathrm{eq})$ in dry toluene $(10 \mathrm{~mL})$, was added PTSA $(24.10 \mathrm{mg}, 0.14 \mathrm{mmol}, 10 \% \mathrm{~mol})$, and the mixture was heated for $24 \mathrm{~h}$ under an inert atmosphere. After evaporating the solvent under reduced pressure, the residue was dissolved in $\mathrm{CH}_{2} \mathrm{Cl}_{2}(50 \mathrm{~mL})$ and then the mixture was vigorously stirred with $\mathrm{Na}_{2} \mathrm{CO}_{3}(0.24 \mathrm{~g}, 8.38 \mathrm{mmol})$ and $\mathrm{H}_{2} \mathrm{O}(1.17$ $\mathrm{mL}, 65 \mathrm{mmol}$ ) for $30 \mathrm{~min}$. The solution was dried over anhydrous $\mathrm{Na}_{2} \mathrm{SO}_{4}$ and filtered. The solvent was evaporated to obtain a pale-yellow solid, which was purified by precipitation from $\mathrm{CH}_{2} \mathrm{Cl}_{2} / \mathrm{Et}_{2} \mathrm{O}$ 7:3 (10 $\left.\mathrm{mL}\right)$ then filtered to afford pure 5a-0. These products could not be purified by absorption chromatography since they extensively decomposed upon contact on silica gel or alumina.

Solvent free microwave irradiation. A mixture of $\alpha$-amino acid phenylhydrazide $3 a-e(2.8 \mathrm{mmol}, 2 \mathrm{eq})$ and the $o$-phthalaldehyde $4 \mathbf{a}$ the $m$-phthalaldehyde $4 \mathbf{b}$ or the $p$-phthalaldehyde $4 \mathrm{c}$ (187.7 $\mathrm{mg}, 1.4 \mathrm{mmol}, 1 \mathrm{eq})$ was submitted to programmed microwave synthesis reactor (START SYNTH) at $100{ }^{\circ} \mathrm{C}$ for $6 \mathrm{~min}$. The crude product was purified by precipitation in a mixture of $\mathrm{CH}_{2} \mathrm{Cl}_{2} / \mathrm{Et}_{2} \mathrm{O} 7: 3(10 \mathrm{~mL})$ in a refrigerator at $3{ }^{\circ} \mathrm{C}$, then filtered to afford pure 5a-o.

(1,2-Phenylene)-2,2'-bis-[5-methyl-3-(phenylamino)imidazolidin-4-one] (5a). White solid, mp 115-117 ${ }^{\circ} \mathrm{C} . \mathrm{R}_{f}$ 0.17 (EtOAc : c- $\mathrm{C}_{6} \mathrm{H}_{12}$ 1:1). IR (neat), $v_{\max }\left(\mathrm{cm}^{-1}\right): 3251,2973,1697,1597,1298 .{ }^{1} \mathrm{H} \mathrm{NMR}\left(300 \mathrm{MHz}, \mathrm{CDCl}_{3}\right): \delta_{\mathrm{H}}$ $1.47\left(6 \mathrm{H}, \mathrm{d},{ }^{3} \mathrm{~J}_{H H} 6.6 \mathrm{~Hz}\right), 2.15(2 \mathrm{H}, \mathrm{s}, \mathrm{NH}), 3.81\left(2 \mathrm{H}, \mathrm{q},{ }^{3} \mathrm{~J}_{H H} 6.6 \mathrm{~Hz}\right), 5.57$ and $5.59(2 \times 1 \mathrm{H}, 2 \mathrm{~s}, \mathrm{CH}), 5.76$ and 5.78 $(2 \times 1 \mathrm{H}, 2 \mathrm{~s}, \mathrm{~N} \underline{\mathrm{HPh}}), 6.55-6.66(4 \mathrm{H}, \mathrm{m}, \mathrm{CH}$ aromatic), 6.85-6.92 (2H, m, CH aromatic), 7.21-7.24 (4H, m, CH aromatic), 7.37-7.42 (4H, m, CH aromatic). $\left.{ }^{13} \mathrm{C} \mathrm{NMR} \mathrm{(75} \mathrm{MHz,} \mathrm{CDCl} 3\right): \delta c 18.22\left(2 \times \mathrm{CH}_{3}\right), 18.49\left(2 \times \mathrm{CH}_{3}\right), 52.61$ $(2 \times \mathrm{CH}), 53.39(2 \times \mathrm{CH}), 74.12(2 \times \mathrm{CH}), 74.70(2 \times \mathrm{CH}), 113.62(4 \times \mathrm{CH}$ aromatic), $113.92(4 \times \mathrm{CH}$ aromatic), 
121.52 ( $2 \times \mathrm{CH}$ aromatic), 121.61 ( $2 \times \mathrm{CH}$ aromatic), 126.18 ( $2 \times \mathrm{CH}$ aromatic), 128.22 ( $2 \times \mathrm{CH}$ aromatic), 128.55 ( $2 \times \mathrm{CH}$ aromatic), 129.26 (4 x CH aromatic), 129.37 (4 × CH aromatic), 129.73 ( $2 \times \mathrm{C}$ aromatic), $138.83(2 \times \mathrm{C}$ aromatic), 139.46 ( $2 \times \mathrm{C}$ aromatic), 145.22 ( $2 \times \mathrm{NHC}$ aromatic), 145.25 ( $2 \times \mathrm{NHC}$ aromatic), 174.45 ( $2 \times \mathrm{C}=0)$, $174.71(2 \times \mathrm{C}=0)$. $\mathrm{ESI}(+)-\mathrm{MS} \mathrm{CH} \mathrm{CN}[\mathrm{C}=0.5, \mathrm{SC}=30, \mathrm{EC}=3] \mathrm{m} / z$ (rel. int.): $457\left(100, \mathrm{M}+\mathrm{H}^{+}\right), 386(20, \mathrm{M}-$ CONHCHMe $+\mathrm{H}^{+}$), 315 (50), 295 (15); HRMS ES ${ }^{+}$for $\mathrm{C}_{26} \mathrm{H}_{29} \mathrm{~N}_{6} \mathrm{O}_{2}[\mathrm{M}+\mathrm{H}]^{+}$Calc. 457.2352, found: 457.2349.

2,2'-(1,2-Phenylene)bis-[5-isopropyl-3-(phenylamino)imidazolidin-4-one] (5b). White solid, mp $112-114{ }^{\circ} \mathrm{C} . \mathrm{R}_{f}$ 0.22 (EtOAc/c- $\mathrm{C}_{6} \mathrm{H}_{12}$ 1:1). IR (neat), $v_{\max }\left(\mathrm{cm}^{-1}\right): 3311,1600,1554,1294 .{ }^{1} \mathrm{H} \mathrm{NMR}\left(300 \mathrm{MHz}, \mathrm{CDCl}_{3}\right): \delta_{\mathrm{H}} 0.89$ $\left(12 \mathrm{H}, \mathrm{d},{ }^{3} \mathrm{~J}_{H H} 7,6 \mathrm{~Hz}\right), 2.02(2 \mathrm{H}, \mathrm{s}, \mathrm{NH}), 2.14-2.17\left(2 \mathrm{H}, \mathrm{m}, \mathrm{CH}\left(\mathrm{CH}_{3}\right)_{2}\right), 3.56\left(2 \mathrm{H}, \mathrm{d},{ }^{3} \mathrm{~J}_{H H} 6.2 \mathrm{~Hz}\right), 6.33$ and $6.36(2 \mathrm{x}$ $1 \mathrm{H}, 2 \mathrm{~s}, \mathrm{CH}), 6.65$ and $6.67(2 \times 1 \mathrm{H}, 2 \mathrm{~s}, \mathrm{NHPh}), 6.91-6.96\left(4 \mathrm{H}, \mathrm{m}, \mathrm{CH}\right.$ aromatic), $7.11\left(2 \mathrm{H}\right.$ aromatic, t, $\left.{ }^{3} J_{H H} 7.4 \mathrm{~Hz}\right)$, $7.16\left(4 \mathrm{H}\right.$ aromatic, d, $\left.{ }^{3} J_{H H} 8.09 \mathrm{~Hz}\right), 7.18\left(2 \mathrm{H}\right.$ aromatic, $\left.d,{ }^{3} \mathrm{HHH}_{\mathrm{HH}} 8.09 \mathrm{~Hz}\right), 7.35-7.38\left(2 \mathrm{H}, \mathrm{m}, \mathrm{CH}\right.$ aromatic). ${ }^{13} \mathrm{C}$ NMR $\left(75 \mathrm{MHz}, \mathrm{CDCl}_{3}\right): \delta_{\mathrm{C}} 20.22\left(2 \times 2 \mathrm{CH}_{3}\right), 32.13\left(\mathrm{CH}\left(\mathrm{CH}_{3}\right)_{2}\right), 32.17\left(\mathrm{CH}\left(\mathrm{CH}_{3}\right)_{2}\right), 74.09(\mathrm{CH}), 74.11(\mathrm{CH}), 80.70(2 \mathrm{x}$ $\mathrm{CHC}=0), 80.73(2 \times \mathrm{CHC}=0), 115.11(2 \times \mathrm{CH}$ aromatic), $117.23(2 \times \mathrm{CH}$ aromatic), 126.55 ( $2 \times \mathrm{CH}$ aromatic), 129.45 ( $2 \times \mathrm{CH}$ aromatic), 131.09 ( $2 \times \mathrm{CH}$ aromatic), 142.16 ( $2 \times \mathrm{C}$ aromatic), 155.01 (2 x NHC aromatic), 174.11 $(2 \times \mathrm{C}=\mathrm{O}), 174.14(2 \times \mathrm{C}=\mathrm{O})$. HRMS ES ${ }^{+}\left(\mathrm{CH}_{3} \mathrm{CN}\right)$ for $\mathrm{C}_{30} \mathrm{H}_{37} \mathrm{~N}_{6} \mathrm{O}_{2} \mathrm{~m} / \mathrm{z}:[\mathrm{M}+\mathrm{H}]^{+}$Calc. 513.2911, found: 513.2914 .

2,2'-(1,2-Phenylene)bis-[5-isobutyl-3-(phenylamino)imidazolidin-4-one] (5c). White solid, mp 118-120 ${ }^{\circ} \mathrm{C} . \mathrm{R}_{f}$ 0.30 (EtOAc/c- $\mathrm{C}_{6} \mathrm{H}_{12}$ 1:1). IR (neat), $v_{\max }\left(\mathrm{cm}^{-1}\right): 3280,2955,1700,1601,1496,1246 .{ }^{1} \mathrm{H} \mathrm{NMR}\left(300 \mathrm{MHz}, \mathrm{CDCl}_{3}\right)$ : $\delta_{H} 0.98\left(6 \mathrm{H}, \mathrm{d},{ }^{3} J_{H H} 6.3 \mathrm{~Hz}\right), 1.04\left(6 \mathrm{H}, \mathrm{d},{ }^{3} J_{H H} 6.6 \mathrm{~Hz}\right), 1.47-1.51\left(2 \mathrm{H}, \mathrm{m}, \mathrm{CH}\left(\mathrm{CH}_{3}\right)_{2}\right), 1.75-1.92\left(4 \mathrm{H}, \mathrm{m}, \mathrm{CH}_{2}\right), 2.19$ $(2 \mathrm{H}, \mathrm{s}, \mathrm{NH}), 5.59\left(2 \mathrm{H}, \mathrm{t},{ }^{3} \mathrm{~J}_{\mathrm{HH}} 6.6 \mathrm{~Hz}\right), 6.53$ and $6.56(2 \times 1 \mathrm{H}, 2 \mathrm{~s}, \mathrm{CH}), 6.59$ and $6.62(2 \times 1 \mathrm{H}, 2 \mathrm{~s}, \mathrm{NHPh}), 6.63-6.69$ $\left(4 \mathrm{H}, \mathrm{m}, \mathrm{CH}\right.$ aromatic), $6.90\left(2 \mathrm{H}, \mathrm{t},{ }^{3} \mathrm{H}_{\mathrm{HH}} 8.1 \mathrm{~Hz}\right), 7.12-7.24(4 \mathrm{H}, \mathrm{m}, \mathrm{CH}$ aromatic), 7.37-7.46 $(4 \mathrm{H}, \mathrm{m}, \mathrm{CH}$ aromatic). ${ }^{13} \mathrm{C} \mathrm{NMR}\left(75 \mathrm{MHz}, \mathrm{CDCl}_{3}\right): \delta \mathrm{C} 21.45\left(2 \times \mathrm{CH}_{3}\right), 23.44\left(2 \times \mathrm{CH}_{3}\right), 29.71(2 \times \mathrm{CH}), 41.81\left(\mathrm{CH}_{2}\right), 41.90\left(\mathrm{CH}_{2}\right), 55.27$ $(\mathrm{CH}), 55.35(\mathrm{CH}), 74.52(2 \times \mathrm{CH}), 74.80(2 \times \mathrm{CH}), 113.63(2 \times \mathrm{CH}$ aromatic), $121.59(2 \times \mathrm{CH}$ aromatic), $126.35(2 \times$ $\mathrm{CH}$ aromatic), 127.14 ( $2 \times \mathrm{CH}$ aromatic), $129.53(2 \times \mathrm{CH}$ aromatic), 138.56 ( $2 \times \mathrm{C}$ aromatic), $145.22(2 \times \mathrm{NHC}$ aromatic), $174.77(2 \times \mathrm{C}=\mathrm{O}), 174.84(2 \times \mathrm{C}=\mathrm{O}) . \mathrm{ESI}(+)-\mathrm{MS} \mathrm{CH}_{3} \mathrm{CN}[\mathrm{C}=0.5, \mathrm{SC}=30, \mathrm{EC}=3] \mathrm{m} / z$ (rel. int.): 541 (50, $\left.\mathrm{M}+\mathrm{H}^{+}\right), 428\left(35, \mathrm{M}-\mathrm{COCHiBuNH}+\mathrm{H}^{+}\right), 315(100), 149$ (55). HRMS ES ${ }^{+}$for $\mathrm{C}_{32} \mathrm{H}_{41} \mathrm{~N}_{6} \mathrm{O}_{2} \mathrm{~m} / \mathrm{z}:[\mathrm{M}+\mathrm{H}]^{+}$Calc. 541.3291, found: 541.3296 .

2,2'-(1,2-Phenylene)bis-[5-benzyl-3-(phenylamino)imidazolidin-4-one] (5d). White solid, mp 124-126 ${ }^{\circ} \mathrm{C} . \mathrm{R}_{f}$ 0.29 (EtOAc/c- $\mathrm{C}_{6} \mathrm{H}_{12}$ 1:1). IR (neat), $v_{\max }\left(\mathrm{cm}^{-1}\right): 3255,1623,1354,1237 .{ }^{1} \mathrm{H}$ NMR $\left(300 \mathrm{MHz}, \mathrm{CDCl}_{3}\right): \delta_{\mathrm{H}} 1.95(2 \mathrm{H}$, $\mathrm{s}, \mathrm{NH}), 3.22\left(2 \mathrm{H}, \mathrm{dd},{ }^{3} J_{H H} 12.3,{ }^{3} J_{H H} 6.00 \mathrm{~Hz}\right), 3.41\left(2 \mathrm{H}, \mathrm{dd},{ }^{3} J_{H H} 12.3,{ }^{3} J_{H H} 6.00 \mathrm{~Hz}\right), 4.00\left(2 \mathrm{H}, \mathrm{t},{ }^{3} J_{H H} 6.00 \mathrm{~Hz}\right), 6.27$ and $6.30(2 \times 1 \mathrm{H}, 2 \mathrm{~s}, \mathrm{CH}), 6.48$ and $6.50(2 \times 1 \mathrm{H}, 2 \mathrm{~s}, \mathrm{NHPh}), 6.79-6.97(4 \mathrm{H}, \mathrm{m}, \mathrm{CH}$ aromatic), 7.05-7.20 $(4 \mathrm{H}, \mathrm{m}$, $\mathrm{CH}$ aromatic), 7.23-7.29 (8H, m, 4CH aromatic), 7.37-7.39 (8H, m, $4 \mathrm{CH}$ aromatic). ${ }^{13} \mathrm{C} \mathrm{NMR}\left(75 \mathrm{MHz}, \mathrm{CDCl}_{3}\right): \delta_{c}$ $37.66\left(\mathrm{CH}_{2}\right), 37.68\left(\mathrm{CH}_{2}\right), 74.22(2 \times \mathrm{CHC}=0), 74.25(2 \times \mathrm{CHC}=0), 77.44(2 \times \mathrm{CH}), 77.47(2 \times \mathrm{CH}), 113.51(2 \times \mathrm{CH}$ aromatic), $113.57(2 \times \mathrm{CH}$ aromatic), $114.04(2 \times \mathrm{CH}$ aromatic), $121.51(2 \times \mathrm{CH}$ aromatic), $125.42(2 \times \mathrm{CH}$ aromatic), 127.17 ( $2 \times \mathrm{CH}$ aromatic), 128.82 ( $\mathrm{CH}$ aromatic), 128.88 ( $\mathrm{CH}$ aromatic), 129.23 (2 x CH aromatic), 129.86 ( $2 \times \mathrm{CH}$ aromatic), 130.09 ( $2 \times \mathrm{CH}$ aromatic), 136.28 ( $2 \times \mathrm{C}$ aromatic), 139.83 ( $2 \times \mathrm{C}$ aromatic), 144.95 (2 x NHC aromatic), 172,69 (2 x C=0). ESI(+)- $\mathrm{MS} \mathrm{CH}_{3} \mathrm{CN}[\mathrm{C}=0.5, \mathrm{SC}=30, \mathrm{EC}=3] \mathrm{m} / z$ (rel. int.): $609\left(100, \mathrm{M}+\mathrm{H}^{+}\right)$, $462\left(40, \mathrm{M}-\mathrm{CONHCHBn}+\mathrm{H}^{+}\right), 315$ (40), 120 (60). $\mathrm{HRMS} \mathrm{ES}^{+}$for $\mathrm{C}_{38} \mathrm{H}_{37} \mathrm{~N}_{6} \mathrm{O}_{2} \mathrm{~m} / \mathrm{z}$ : $[\mathrm{M}+\mathrm{H}]^{+}$Calc. 609.2978, found: 609.2976.

2,2'-(1,2-Phenylene)bis-[5-(2-methylthioethyl)-3-(phenylamino)imidazolidin-4-one] (5e). White solid, $\mathrm{mp}$ 123-125 ${ }^{\circ} \mathrm{C} . \mathrm{R}_{f} 0.45$ (EtOAc/c- $\mathrm{C}_{6} \mathrm{H}_{12}$ 1:1). IR (neat), $v_{\max }\left(\mathrm{cm}^{-1}\right): 3275,1690,1367,1278 .{ }^{1} \mathrm{H} \mathrm{NMR}(300 \mathrm{MHz}$, $\left.\mathrm{CDCl}_{3}\right): \delta_{\mathrm{H}} 1.91-2.15(2 \mathrm{H}, \mathrm{m}, \mathrm{CH}), 2.16\left(6 \mathrm{H}, \mathrm{s}, \mathrm{SCH}_{3}\right), 2.19-2.38(2 \mathrm{H}, \mathrm{m}, \mathrm{CH}), 2.62(2 \mathrm{H}, \mathrm{s}, \mathrm{NH}), 2.75-2.79(4 \mathrm{H}, \mathrm{m}$, $\left.\mathrm{CH}_{2}\right), 3.96-4.01(2 \mathrm{H}, \mathrm{m}, \mathrm{CHC}=\mathrm{O}), 5.56$ and $5.60(2 \times 1 \mathrm{H}, 2 \mathrm{~s}, \mathrm{CH}), 5.63$ and $5.83(2 \times 1 \mathrm{H}, 2 \mathrm{~s}, \mathrm{NHPh}), 6.57(2 \mathrm{H}, \mathrm{d}$, $\left.{ }^{3} J_{H H} 8.1 \mathrm{~Hz}\right), 6.65\left(2 \mathrm{H}, \mathrm{d},{ }^{3} \mathrm{~J}_{H H} 7.8 \mathrm{~Hz}\right), 6.86-6.94(2 \mathrm{H}, \mathrm{m}, \mathrm{CH}$ aromatic), 7.13-7.23 $(4 \mathrm{H}, \mathrm{m}, \mathrm{CH}$ aromatic), 7.33-7.51 $\left(4 \mathrm{H}, \mathrm{m}, \mathrm{CH}\right.$ aromatic). ${ }^{13} \mathrm{C} \mathrm{NMR}\left(75 \mathrm{MHz}, \mathrm{CDCl}_{3}\right): \delta_{\mathrm{C}} 15.31\left(\mathrm{CH}_{2}\right), 15.37,\left(\mathrm{CH}_{2}\right), 30.35\left(\mathrm{SCH}_{3}\right), 31.48\left(\mathrm{CH}_{2}\right), 31.67$ $\left(\mathrm{CH}_{2}\right), 55.65(2 \times \mathrm{CH}), 56.13(2 \times \mathrm{CH}), 74.38(2 \times \mathrm{CH}), 74.82(2 \times \mathrm{CH}), 113.69(2 \times \mathrm{CH}$ aromatic $), 113.84(2 \times \mathrm{CH}$ 
aromatic), 121.62 (2 $\times \mathrm{CH}$ aromatic), 122.28 ( $\mathrm{CH}$ aromatic), 126.21 ( $\mathrm{CH}$ aromatic), 129.27 ( $2 \times \mathrm{CH}$ aromatic), 139.12 (C aromatic), 145.25 ( $2 \times \mathrm{NHC}$ aromatic), $173.36(2 \times \mathrm{C}=0), 173.73(2 \times \mathrm{C}=0) . \mathrm{ESI}(+)-\mathrm{MS} \mathrm{CH}_{3} \mathrm{CN}[\mathrm{C}=0.5$, $\mathrm{SC}=30, \mathrm{EC}=3] \mathrm{m} / z$ (rel. int.): $577\left(100, \mathrm{M}+\mathrm{H}^{+}\right), 446\left(5, \mathrm{M}-\mathrm{CONHCH}\left(\mathrm{CH}_{2}\right)_{2} \mathrm{SMe}+\mathrm{H}^{+}\right), 355$ (10). HRMS ES ${ }^{+}$for $\mathrm{C}_{30} \mathrm{H}_{37} \mathrm{~N}_{6} \mathrm{O}_{2} \mathrm{~S}_{2} \mathrm{~m} / \mathrm{z}$ : $[\mathrm{M}+\mathrm{H}]^{+}$Calc. 577.2419, found: 577.2416 .

2,2'-(1,3-Phenylene)bis-[5-methyl-3-(phenylamino)imidazolidin-4-one] (5f). White solid, mp 116-118 ${ }^{\circ} \mathrm{C} . \mathrm{R}_{f}$ 0.18 (EtOAc/c- $\mathrm{C}_{6} \mathrm{H}_{12}$ 1:1). IR (neat), $v_{\max }\left(\mathrm{cm}^{-1}\right): 3253,3024,1707,1694,1600,1495 .{ }^{1} \mathrm{H} \mathrm{NMR}\left(300 \mathrm{MHz}, \mathrm{CDCl}_{3}\right)$ : $\delta_{H} 1.52$ and $1.54\left(6 \mathrm{H}, 2 \mathrm{~d},{ }^{3} \mathrm{~J}_{H H} 6.6 \mathrm{~Hz}\right), 1.80\left(2 \mathrm{H}\right.$, broad s, NH), $3.81\left(2 \mathrm{H}, \mathrm{q},{ }^{3} \mathrm{~J}_{\mathrm{HH}} 6.6 \mathrm{~Hz}\right), 5.55$ and $5.58(2 \times 1 \mathrm{H}, 2 \mathrm{~s}$, $\mathrm{CH}), 5.76$ and $5.78(2 \times 1 \mathrm{H}, 2 \mathrm{~s}, \mathrm{NHPh}), 6.55-6.66(4 \mathrm{H}, \mathrm{m}, \mathrm{CH}$ aromatic), 7.11-7.15 (2H, m, CH aromatic), 7.21$7.24\left(4 \mathrm{H}, \mathrm{m}, \mathrm{CH}\right.$ aromatic), 7.37-7.42 (4H, $\mathrm{m}, \mathrm{CH}$ aromatic). ${ }^{13} \mathrm{C} \mathrm{NMR}\left(75 \mathrm{MHz}, \mathrm{CDCl}_{3}\right): \delta \mathrm{c} 18.29\left(2 \times \mathrm{CH}_{3}\right), 18.50$ $\left(2 \times \mathrm{CH}_{3}\right), 52.67(2 \times \mathrm{CH}), 53.35(2 \times \mathrm{CH}), 73.97(2 \times \mathrm{CH}), 74.40(2 \times \mathrm{CH}), 113.74(4 \times \mathrm{CH}$ aromatic), $113.98(4 \times \mathrm{CH}$ aromatic), 121.61 ( $4 \times \mathrm{CH}$ aromatic), 121.75 (4 $\times$ CH aromatic), 127.57 ( $2 \times \mathrm{CH}$ aromatic), $128.41(2 \times \mathrm{CH}$ aromatic), 129.25 (4 x CH aromatic), $129.38(4 \times \mathrm{CH}$ aromatic), 139.05 ( $2 \times \mathrm{CH}$ aromatic), $140.09(2 \times \mathrm{C}$ aromatic), 145.08 ( $2 \times \mathrm{NHC}$ aromatic), 145.24 ( $2 \times \mathrm{NHC}$ aromatic), 174.48 ( $2 \times \mathrm{C}=0), 174.55$ ( $2 \times \mathrm{C}=0)$. ESI(+)-MS $\mathrm{CH}_{3} \mathrm{CN} m / z$ (rel. int.): 530 (15), $495\left(10, \mathrm{M}+\mathrm{K}^{+}\right), 479\left(60, \mathrm{M}+\mathrm{Na}^{+}\right), 457\left(100, \mathrm{M}+\mathrm{H}^{+}\right)$. HRMS ES for $\mathrm{C}_{26} \mathrm{H}_{29} \mathrm{~N}_{6} \mathrm{O}_{2}$ $\mathrm{m} / \mathrm{z}:[\mathrm{M}+\mathrm{H}]^{+}$Calc. 457.2347 , found: 457.2342 .

2,2'-(1,3-Phenylene)bis-[5-isopropyl-3-(phenylamino)imidazolidin-4-one] (5g). White solid, mp 111-113 ${ }^{\circ} \mathrm{C} . \mathrm{R}_{f}$ 0.21 (EtOAc/c- $C_{6} \mathrm{H}_{12}$ 1:1). IR (neat), $v_{\max }\left(\mathrm{cm}^{-1}\right): 3300,1620,1543,1290 .{ }^{1} \mathrm{H} N M R\left(300 \mathrm{MHz}, \mathrm{CDCl}_{3}\right): \delta_{\mathrm{H}} 0.91$ $\left(12 \mathrm{H}, \mathrm{d},{ }^{3} \mathrm{~J}_{\mathrm{HH}} 7.4 \mathrm{~Hz}\right), 2.00(2 \mathrm{H}, \mathrm{s}, \mathrm{NH}), 2.16-2.18\left(2 \mathrm{H}, \mathrm{m}, \mathrm{CH}\left(\mathrm{CH}_{3}\right)_{2}\right), 3.74\left(2 \mathrm{H}, \mathrm{d},{ }^{3} \mathrm{~J}_{H H} 6.2 \mathrm{~Hz}\right), 6.31$ and $6.33(2 \mathrm{x}$ $1 \mathrm{H}, 2 \mathrm{~s}, \mathrm{CH}), 6.55$ and $6.57(2 \times 1 \mathrm{H}, 2 \mathrm{~s}, \mathrm{NHPh}), 6.87-6.90\left(4 \mathrm{H}, \mathrm{m}, \mathrm{CH}\right.$ aromatic), $7.09\left(2 \mathrm{H}, \mathrm{t},{ }^{3} \mathrm{JHH}_{\mathrm{HH}} 7.9 \mathrm{~Hz}\right), 7.14(4 \mathrm{H}$, d, $\left.{ }^{3} J_{H H} 8.1 \mathrm{~Hz}\right), 7.18\left(2 \mathrm{H}, \mathrm{d},{ }^{3} J_{H H} 8.1 \mathrm{~Hz}\right), 7.32-7.36\left(2 \mathrm{H}, \mathrm{m}, \mathrm{CH}\right.$ aromatic). ${ }^{13} \mathrm{C} \mathrm{NMR}\left(75 \mathrm{MHz}, \mathrm{CDCl}_{3}\right): \delta_{\mathrm{C}} 20.44$ $\left(\left(\mathrm{CH}_{3}\right)_{2}\right), 31.23(\mathrm{CH}), 31.25(\mathrm{CH}), 73.19(\mathrm{CH}), 73.21(\mathrm{CH}), 83.73(2 \times \mathrm{CH}), 83.75(2 \times \mathrm{CH}), 115.13(2 \times \mathrm{CH}$ aromatic), 116.25 ( $2 \times \mathrm{CH}$ aromatic), $126.45(2 \times \mathrm{CH}$ aromatic), $128.62(2 \times \mathrm{CH}$ aromatic), $130.15(2 \times \mathrm{CH}$ aromatic), 131.11 ( $2 \times \mathrm{CH}$ aromatic), 142.54 ( $2 \times \mathrm{C}$ aromatic), 156.41 ( $2 \times \mathrm{NHC}$ aromatic), $174.41(2 \times \mathrm{C}=\mathrm{O})$, $174.43(2 \times \mathrm{C}=0)$.

2,2'-(1,3-Phenylene)bis-[5-isobutyl-3-(phenylamino)imidazolidin-4-one] (5h). White solid, mp 119-121 ${ }^{\circ} \mathrm{C} . \mathrm{R}_{f}$ 0.28 (EtOAc/c- $\mathrm{C}_{6} \mathrm{H}_{12}$ 1:1). IR (neat), $v_{\max }\left(\mathrm{cm}^{-1}\right): 3032,2954,2628,1579,1512,1405 .{ }^{1} \mathrm{H}-\mathrm{NMR}\left(300 \mathrm{MHz}, \mathrm{CDCl}_{3}\right)$ : $\delta=1.00\left(6 \mathrm{H}, \mathrm{d},{ }^{3} J_{H H} 5.7 \mathrm{~Hz}\right), 1.04\left(6 \mathrm{H}, \mathrm{d},{ }^{3} \mathrm{~J}_{H H} 6.3 \mathrm{~Hz}\right), 1.56-1.62\left(2 \mathrm{H}, \mathrm{m}, \mathrm{CH}\left(\mathrm{CH}_{3}\right)_{2}\right), 1.88-1.90\left(4 \mathrm{H}, \mathrm{m}, \mathrm{CH}_{2}\right), 2.18$ $(2 \mathrm{H}, \mathrm{s}, \mathrm{NH}), 3.85\left(2 \mathrm{H}, \mathrm{dd},{ }^{3} \mathrm{~J}_{\mathrm{HH}} 14.4,{ }^{3} \mathrm{~J}_{\mathrm{HH}} 8.4 \mathrm{~Hz}\right), 5.61$ and $5.63(2 \times 1 \mathrm{H}, 2 \mathrm{~s}, \mathrm{CH}), 5.69$ and $5.71(2 \times 1 \mathrm{H}, 2 \mathrm{~s}, \mathrm{NHPh})$, 6.61-6.70 (4H, m, CH aromatic), 6.88-6.95 (2H, m, CH aromatic), 7.16-7.28 (4H, m, CH aromatic), 7.29-7.45 (4H, m, CH aromatic). ${ }^{13} \mathrm{C} \mathrm{NMR}\left(75 \mathrm{MHz}, \mathrm{CDCl}_{3}\right): \delta_{\mathrm{C}} 21.45\left(4 \times \mathrm{CH}_{3}\right), 21.47\left(4 \times \mathrm{CH}_{3}\right), 25.08(2 \times \mathrm{CH}), 42.03\left(2 \times \mathrm{CH}_{2}\right)$, $42.07\left(2 \times \mathrm{CH}_{2}\right), 55.29(2 \times \mathrm{CH}), 55.91(2 \times \mathrm{CH}), 74.21(2 \times \mathrm{CH}), 74.58(2 \times \mathrm{CH}), 113.75(4 \times \mathrm{CH}$ aromatic), 114.00 (4 $\times$ CH aromatic), 121.57 ( $4 \times \mathrm{CH}$ aromatic), 121.69 ( $4 \times \mathrm{CH}$ aromatic), 127.62 ( $2 \times \mathrm{CH}$ aromatic), $128.49(2 \times \mathrm{CH}$ aromatic), 129.23 ( $4 \times \mathrm{CH}$ aromatic), 129.36 ( $4 \times \mathrm{CH}$ aromatic), 140.22 ( $2 \times \mathrm{C}$ aromatic), $145.14(2 \times \mathrm{NHC}$ aromatic), 145.26 ( $2 \times \mathrm{NHC}$ aromatic), $174.44(2 \times \mathrm{C}=0), 174.68$ ( $2 \times \mathrm{C}=0)$. ESI(+)-MS CH${ }_{3} \mathrm{CN} \mathrm{m/z}$ (rel. int.): 579 $\left(15, \mathrm{M}+\mathrm{K}^{+}\right), 563\left(100, \mathrm{M}+\mathrm{Na}^{+}\right), 541\left(75, \mathrm{M}+\mathrm{H}^{+}\right), 464\left(15, \mathrm{M}-\mathrm{Ph}+\mathrm{H}^{+}\right), 453$ (55), 413 (10), 391 (15). HRMS ES for $\mathrm{C}_{30} \mathrm{H}_{41} \mathrm{~N}_{6} \mathrm{O}_{2} \mathrm{~m} / \mathrm{z}$ : [M+H] ${ }^{+}$Calc. 541.3286, found: 541.3282 .

2,2'-(1,3-Phenylene)bis-[5-benzyl-3-(phenylamino)imidazolidin-4-one] (5i). White solid, $\mathrm{mp} 127-129{ }^{\circ} \mathrm{C} . \mathrm{R}_{f}$ 0.29 (EtOAc/c- $\mathrm{C}_{6} \mathrm{H}_{12}$ 1:1). IR (neat), $v_{\max }\left(\mathrm{cm}^{-1}\right): 3294,2920,1699,1601,1495,1453,1090 .{ }^{1} \mathrm{H}-\mathrm{NMR}(300 \mathrm{MHz}$, $\left.\mathrm{CDCl}_{3}\right): \delta_{\mathrm{H}} 1.14(2 \mathrm{H}, \mathrm{s}, \mathrm{NH}), 3.10-3.30\left(4 \mathrm{H}, \mathrm{m}, \mathrm{CH}_{2}\right), 3.99-4.09(2 \mathrm{H}, \mathrm{m}, \mathrm{CH}), 5.20$ and $5.27(2 \times 1 \mathrm{H}, 2 \mathrm{~s}, \mathrm{CH}), 5.43$ and $5.48(2 \times 1 \mathrm{H}, 2 \mathrm{~s}, \mathrm{NHPh}), 6.18-6.27(2 \mathrm{H}, \mathrm{m}, \mathrm{CH}$ aromatic), 6.41-6.44 (2H, m, CH aromatic), 6.64-6.68 (2H, $\mathrm{m}$, $\mathrm{CH}$ aromatic), 6.78-6.80 (4H, $\mathrm{m}, \mathrm{CH}$ aromatic); 7.00-7.07 (6H, $\mathrm{m}, \mathrm{CH}$ aromatic), 7.19-7.29 (8H, $\mathrm{m}, \mathrm{CH}$ aromatic). ${ }^{13} \mathrm{C}-\mathrm{NMR}\left(75 \mathrm{MHz}, \mathrm{CDCl}_{3}\right):$ c $37.98\left(\mathrm{CH}_{2}\right), 38.01\left(\mathrm{CH}_{2}\right), 55.23(2 \times \mathrm{CH}), 55.25(2 \times \mathrm{CH}), 77.01(2 \times \mathrm{CH}), 77.03(2 \times$ $\mathrm{CH}), 113.11$ ( $2 \times \mathrm{CH}$ aromatic), $115.02(2 \times \mathrm{CH}$ aromatic), $122.12(2 \times \mathrm{CH}$ aromatic), 125.55 (2 $\mathrm{CH}$ aromatic), 127.56 ( $2 \times \mathrm{CH}$ aromatic), 128.11 ( $2 \times \mathrm{CH}$ aromatic), 128.61 ( $2 \times \mathrm{CH}$ aromatic), 129.21 (2 $\times \mathrm{CH}$ aromatic), 129.45 
(CH aromatic), 131.13 ( $\mathrm{CH}$ aromatic), $137.33(2 \times \mathrm{C}$ aromatic), 139.23 ( $2 \times \mathrm{C}$ aromatic), $145.54(2 \times \mathrm{NHC}$

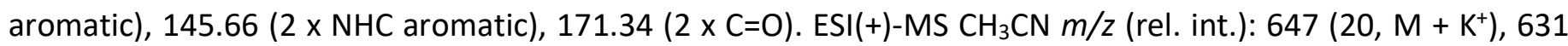
(95, M + Na+), $609\left(100, M+\mathrm{H}^{+}\right), 566$ (15), 453 (30), 343 (10, M-imidazolidinone + $\mathrm{H}^{+}$), 294 (25), 272 (35). HRMS ES ${ }^{+}$for $\mathrm{C}_{38} \mathrm{H}_{37} \mathrm{~N}_{6} \mathrm{O}_{2} \mathrm{~m} / \mathrm{z}$ : [M+H] ${ }^{+}$Calc. 609.2973, found: 609.2966.

2,2'-(1,3-Phenylene)bis-[5-(2-methylthioethyl)-3-(phenylamino)imidazolidin-4-one] (5j). White solid, mp 124$126{ }^{\circ} \mathrm{C} . \mathrm{R}_{f} 0.46$ (EtOAc/c- $\mathrm{C}_{6} \mathrm{H}_{12}$ 1:1). IR (neat), $v_{\max }\left(\mathrm{cm}^{-1}\right): 3278,2913,1695,1601,1495,1445,1092 .{ }^{1} \mathrm{H}$ NMR (300 MHz, $\left.\mathrm{CDCl}_{3}\right): \delta_{\mathrm{H}}$ 1.96-2.06 $(2 \mathrm{H}, \mathrm{m}, \mathrm{CH}), 2.14\left(6 \mathrm{H}, \mathrm{s}, \mathrm{CH}_{3}\right), 2.31-2.62(2 \mathrm{H}, \mathrm{m}, \mathrm{CH}), 2.74-2.96\left(4 \mathrm{H}, \mathrm{m}, \mathrm{CH}_{2}\right)$, 4.00-4.04 (2H, m, CH), 5.52 and $5.62(2 \times 1 \mathrm{H}, 2 \mathrm{~s}, \mathrm{CH}), 5.64$ and $5.69(2 \times 1 \mathrm{H}, 2 \mathrm{~s}, \mathrm{NHPh}), 6.54-6.61(4 \mathrm{H}, \mathrm{m}, \mathrm{CH}$ aromatic), 6.65-6.71 (2H, $\mathrm{m}, \mathrm{CH}$ aromatic), 7.15-7.20 $(4 \mathrm{H}, \mathrm{m}, \mathrm{CH}$ aromatic), 7.33-7.37 (4H, $\mathrm{m}, \mathrm{CH}$ aromatic), 7.42-7.47 (2H, m, CH aromatic). ${ }^{13} \mathrm{C} \mathrm{NMR}\left(75 \mathrm{MHz}, \mathrm{CDCl}_{3}\right): \delta c 15.27\left(4 \times \mathrm{CH}_{2}\right), 15.36\left(4 \times \mathrm{CH}_{2}\right), 30.34\left(4 \times \mathrm{SCH}_{3}\right)$, $31.51\left(2 \times \mathrm{CH}_{2}\right), 31.59\left(2 \times \mathrm{CH}_{2}\right), 74.40(2 \times \mathrm{CH}), 74.44(2 \times \mathrm{CH}), 77.43(2 \times \mathrm{CH}), 77.50(2 \times \mathrm{CH}), 113.72(4 \times \mathrm{CH}$ aromatic), 113.94 ( $4 \times \mathrm{CH}$ aromatic), $121.66(2 \times \mathrm{CH}$ aromatic), $122.04(2 \times \mathrm{CH}$ aromatic), $125.23(4 \times \mathrm{CH}$

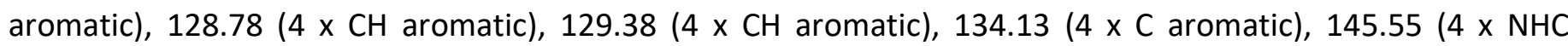
aromatic), 173.31 ( $2 \times \mathrm{C}=0), 173.35(2 \times \mathrm{C}=0)$. $\mathrm{ESI}(+)-\mathrm{MS} \mathrm{CH}_{3} \mathrm{CN} \mathrm{m/z} \mathrm{(rel.} \mathrm{int.):} 615$ (10, $\left.\mathrm{M}+\mathrm{K}^{+}\right), 599$ (70, $\mathrm{M}+$ $\left.\mathrm{Na}^{+}\right), 577\left(100, \mathrm{M}+\mathrm{H}^{+}\right), 453$ (10). $\mathrm{HRMS} \mathrm{ES}^{+}$for $\mathrm{C}_{30} \mathrm{H}_{37} \mathrm{~N}_{6} \mathrm{O}_{2} \mathrm{~S}_{2} \mathrm{~m} / \mathrm{z}$ : [M+H] ${ }^{+}$Calc. 577.2414, found: 577.2408.

2,2'-(1,4-Phenylene)bis-[5-methyl-3-(phenylamino)imidazolidin-4-one] (5k). White solid, mp 117-119 ${ }^{\circ} \mathrm{C} . \mathrm{R}_{f}$ 0.17 (EtOAc/c- $\mathrm{C}_{6} \mathrm{H}_{12}$ 1:1). IR (neat), $v_{\max }\left(\mathrm{cm}^{-1}\right): 3254,3022,1708,1600,1495,1392 .{ }^{1} \mathrm{H} \mathrm{NMR}\left(300 \mathrm{MHz}, \mathrm{CDCl}_{3}\right)$ : $\delta_{\mathrm{H}} 1.44\left(6 \mathrm{H}, \mathrm{d},{ }^{3} \mathrm{~J}_{\mathrm{HH}} 6.9 \mathrm{~Hz}\right), 2.00(2 \mathrm{H}, \mathrm{s}, \mathrm{NH}), 3.74\left(2 \mathrm{H}, \mathrm{q}^{3}{ }^{3} \mathrm{JHH} 6.9 \mathrm{~Hz}\right), 6.00$ and $6.02(2 \times 1 \mathrm{H}, 2 \mathrm{~s}, \mathrm{CH}), 6.70$ and $6.72(2 \times 1 \mathrm{H}, 2 \mathrm{~s}, \mathrm{NHPh}), 6.78\left(4 \mathrm{H}, \mathrm{dd},{ }^{3} \mathrm{~J}_{H H} 7.2,{ }^{3} \mathrm{~J}_{H H} 6.9 \mathrm{~Hz}\right), 6.85-6.92(2 \mathrm{H}, \mathrm{m}, \mathrm{CH}$ aromatic), 7.12-7.22 $(4 \mathrm{H}, \mathrm{m}$, $\mathrm{CH}$ aromatic), 7.34-7.48 (4H, m, CH aromatic). $\left.{ }^{13} \mathrm{C} \mathrm{NMR} \mathrm{(75} \mathrm{MHz,} \mathrm{CDCl} 3\right): \delta c 23.70\left(2 \times \mathrm{CH}_{3}\right), 23.71\left(2 \times \mathrm{CH}_{3}\right)$, $51.81(2 \times \mathrm{CH}), 51.83(2 \times \mathrm{CH}), 75.19(2 \times \mathrm{CH}), 75.21(2 \times \mathrm{CH}), 113.22(4 \times \mathrm{CH}$ aromatic), $113.24(4 \times \mathrm{CH}$ aromatic), 122.82 (4 x CH aromatic), 125.39 (4 x CH aromatic), 125.41 (4 x CH aromatic), $129.26(4 \times \mathrm{CH}$ aromatic), 129.27 (4 x CH aromatic), $137.83(2 \times \mathrm{C}$ aromatic), $137.86(2 \times \mathrm{C}$ aromatic), $151.00(2 \times \mathrm{NHC}$ aromatic), 151.03 ( $2 \times \mathrm{NHC}$ aromatic), $173.41(2 \times \mathrm{C}=0), 173.43(2 \times \mathrm{C}=0) . \mathrm{ESI}(+)-\mathrm{MS} \mathrm{CH} \mathrm{CN}$ [C $=0.5, \mathrm{SC}=30, \mathrm{EC}$ = 3] $\mathrm{m} / \mathrm{z}$ (rel. int.): $935\left(5,2 \mathrm{M}+\mathrm{Na}^{+}\right), 457\left(50, \mathrm{M}+\mathrm{H}^{+}\right), 386\left(40, \mathrm{M}-\mathrm{COCHMeNH}+\mathrm{H}^{+}\right), 315$ (100), 242 (10). HRMS ES ${ }^{+}$for $\mathrm{C}_{26} \mathrm{H}_{29} \mathrm{~N}_{6} \mathrm{O}_{2} \mathrm{~m} / \mathrm{z}$ : $[\mathrm{M}+\mathrm{H}]^{+}$Calc. 457.2352, found: 457.2346 .

2,2'-(1,4-Phenylene)bis-[5-isopropyl-3-(phenylamino)imidazolidin-4-one] (5I). Yellow liquid, mp $113-115{ }^{\circ} \mathrm{C}$. $\mathrm{R}_{f} 0.22$ (EtOAc/c-C $\mathrm{H}_{12}$ 1:1). IR (neat), $v_{\max }\left(\mathrm{cm}^{-1}\right): 3330,1601,1555,1300 .{ }^{1} \mathrm{H}$ NMR $\left(300 \mathrm{MHz}, \mathrm{CDCl}_{3}\right): \delta_{\mathrm{H}} 1.00$ $\left(12 \mathrm{H}, \mathrm{d},{ }^{3} \mathrm{~J}_{H H} 6.2 \mathrm{~Hz}\right), 1.92(2 \mathrm{H}, \mathrm{s}, \mathrm{NH}), 2.28-2.31(2 \mathrm{H}, \mathrm{m}, \mathrm{CH}), 3.65\left(2 \mathrm{H}, \mathrm{d},{ }^{3} \mathrm{~J}_{H H} 7.4 \mathrm{~Hz}\right), 6.00$ and $6.02(2 \times 1 \mathrm{H}, 2 \mathrm{~s}$, $\mathrm{CH}), 6.62$ and $6.64(2 \times 1 \mathrm{H}, 2 \mathrm{~s}, \mathrm{NHPh}), 7.24-7.26\left(4 \mathrm{H}, \mathrm{m}, \mathrm{CH}\right.$ aromatic), 7.38-7.41 (10H, m, CH aromatic). ${ }^{13} \mathrm{C}$ $\operatorname{NMR}\left(75 \mathrm{MHz}, \mathrm{CDCl}_{3}\right): \delta \mathrm{c} 20.15\left(2 \times\left(\mathrm{CH}_{3}\right)_{2}\right), 20.19\left(2 \times\left(\mathrm{CH}_{3}\right)_{2}\right), 33.00(\mathrm{CH}), 33.04(\mathrm{CH}), 74.60(\mathrm{CH}), 74.66(\mathrm{CH})$, $75.20(2 \times \mathrm{CH}), 75.24(2 \times \mathrm{CH}), 113.30(2 \times \mathrm{CH}$ aromatic), 122.02 ( $\mathrm{CH}$ aromatic), 126.00 ( $\mathrm{CH}$ aromatic), $127.00(2$ x CH aromatic), 130.10 ( $2 \times$ C aromatic), 151.30 ( $2 \times$ NHC aromatic), 173.30 ( $2 \times \mathrm{C}=0), 173.32$ ( $2 \times \mathrm{C}=0)$.

2,2'-(1,4-Phenylene)bis-[5-isobutyl-3-(phenylamino)imidazolidin-4-one] (5m). White solid, mp $120-122^{\circ} \mathrm{C} . \mathrm{R}_{f}$ 0.33 (EtOAc/c- $\mathrm{C}_{6} \mathrm{H}_{12}$ 1:1). IR (neat), $v_{\max }\left(\mathrm{cm}^{-1}\right): 3263,2957,1703,1602,1497,1082 .{ }^{1} \mathrm{H} \mathrm{NMR}\left(300 \mathrm{MHz}, \mathrm{CDCl}_{3}\right)$ : $\delta_{H} 1.01\left(6 \mathrm{H}, \mathrm{d}, 3^{3} J_{H H} 6.4 \mathrm{~Hz}\right), 1.05\left(6 \mathrm{H}, \mathrm{d},{ }^{3} J_{H H} 6.7 \mathrm{~Hz}\right), 1.55-1.59(2 \mathrm{H}, \mathrm{m}, \mathrm{CH}), 1.88-1.90\left(4 \mathrm{H}, \mathrm{m}, \mathrm{CH}_{2}\right), 3.82-3.85$ $(2 \mathrm{H}, \mathrm{m}, \mathrm{CH}), 5.59(2 \mathrm{H}, \mathrm{s}, \mathrm{CH}), 5.61(2 \mathrm{H}, \mathrm{s}, \mathrm{NH}), 6.87-6.92\left(4 \mathrm{H}, \mathrm{m}, \mathrm{CH}\right.$ aromatic), $7.05\left(4 \mathrm{H}, \mathrm{d}, 3_{\mathrm{HH}} 8.7 \mathrm{~Hz}\right), 7.08-$ $7.11\left(4 \mathrm{H}, \mathrm{m}, \mathrm{CH}\right.$ aromatic), 7.33-7.37 (4H, m, CH aromatic). ${ }^{13} \mathrm{C} \mathrm{NMR}\left(75 \mathrm{MHz}, \mathrm{CDCl}_{3}\right)$ : $\delta_{\mathrm{C}} 21.51\left(\mathrm{CH}_{3}\right), 23.36$ $\left(\mathrm{CH}_{3}\right), 25.09(2 \times \mathrm{CH}), 42.04\left(\mathrm{CH}_{2}\right), 42.10\left(\mathrm{CH}_{2}\right), 55.29(\mathrm{CH}), 55.92(\mathrm{CH}), 74.26(\mathrm{CH}), 74.65(\mathrm{CH}), 113.81(2 \times \mathrm{CH}$ aromatic), $121.59(2 \times \mathrm{CH}$ aromatic), $121.70(2 \times \mathrm{CH}$ aromatic), $127.59(2 \times \mathrm{CH}$ aromatic), $127.68(2 \times \mathrm{CH}$ aromatic), 129.20 ( $2 \times \mathrm{CH}$ aromatic), 129.33 ( $2 \times \mathrm{CH}$ aromatic), 140.25 ( $2 \times \mathrm{C}$ aromatic), 145.20 (NHC aromatic), 145.32 (NHC aromatic), $174.39(2 \times \mathrm{C}=0), 174.64(2 \times \mathrm{C}=0) . \mathrm{ESI}(+)-\mathrm{MS} \mathrm{CH} \mathrm{CN}_{3}[\mathrm{C}=2, \mathrm{SC}=20, \mathrm{EC}=2] \mathrm{m} / \mathrm{z}$ (rel. int.): 631 (15), $563\left(100, \mathrm{M}+\mathrm{Na}^{+}\right), 541\left(90, \mathrm{M}+\mathrm{H}^{+}\right), 428\left(10, \mathrm{M}-\mathrm{COCHiBuNH}+\mathrm{H}^{+}\right), 301$ (15), 242 (25). HRMS $\mathrm{ES}^{+}$for $\mathrm{C}_{32} \mathrm{H}_{41} \mathrm{~N}_{6} \mathrm{O}_{2} \mathrm{~m} / \mathrm{z}$ : $[\mathrm{M}+\mathrm{H}]^{+}$Calc. 541.3291, found: 541.3293 . 
2,2'-(1,4-Phenylene)bis-[5-benzyl-3-(phenylamino)imidazolidin-4-one] (5n). White solid, mp $129-131^{\circ} \mathrm{C} . \mathrm{R}_{f}$ 0.28 (EtOAc/c- $\mathrm{C}_{6} \mathrm{H}_{12}$ 1:1). IR (neat), $v_{\max }\left(\mathrm{cm}^{-1}\right): 3277,3031,1699,1590,1398 .{ }^{1} \mathrm{H}-\mathrm{NMR}\left(300 \mathrm{MHz}, \mathrm{CDCl}_{3}\right): \delta_{\mathrm{H}}$ $1.28(2 \mathrm{H}, \mathrm{s}, \mathrm{NH}), 3.41\left(2 \mathrm{H}, \mathrm{dd},{ }^{3} J_{H H} 12.3,{ }^{3} J_{H H} 6.0 \mathrm{~Hz}\right), 3.55\left(2 \mathrm{H}, \mathrm{dd},{ }^{3} J_{H H} 12.3,{ }^{3} J_{H H} 6.0 \mathrm{~Hz}\right), 4.02\left(2 \mathrm{H}, \mathrm{t}, 3^{3} J_{H H} 6.0\right.$ $\mathrm{Hz}), 4.09\left(2 \mathrm{H}, \mathrm{t},{ }^{3} \mathrm{~J}_{\mathrm{HH}} 6.0 \mathrm{~Hz}\right), 6.27$ and $6.30(2 \times 1 \mathrm{H}, 2 \mathrm{~s}, \mathrm{CH}), 6.48$ and $6.50(2 \times 1 \mathrm{H}, 2 \mathrm{~s}, \mathrm{NHPh}), 6.79-6.97(8 \mathrm{H}, \mathrm{m}$, $\mathrm{CH}$ aromatic), 7.05-7.20 (3H, $\mathrm{m}, \mathrm{CH}$ aromatic), 7.23-7.29 (8H, $\mathrm{m}, \mathrm{CH}$ aromatic), 7.37-7.39 (3H, $\mathrm{m}, \mathrm{CH}$ aromatic). ${ }^{13} \mathrm{C} \mathrm{NMR}\left(75 \mathrm{MHz}, \mathrm{CDCl}_{3}\right): \delta \mathrm{c} 36.84\left(\mathrm{CH}_{2}\right), 57.74(\mathrm{CH}), 58.40(\mathrm{CH}), 73.88(2 \times \mathrm{CH}), 74.83(2 \times \mathrm{CH}), 113.66(4 \times \mathrm{CH}$ aromatic), 114.07 ( $2 \times \mathrm{CH}$ aromatic), 121.51 ( $2 \times \mathrm{CH}$ aromatic), 125.42 ( $\mathrm{CH}$ aromatic), 127.17 ( $2 \times \mathrm{CH}$ aromatic), 129.11 (4 x CH aromatic), 129.23 (4 x CH aromatic), 130.09 ( $2 \times \mathrm{CH}$ aromatic), 136.28 (2 $\times \mathrm{CH}$ aromatic), 139.83 ( $2 \times$ C aromatic), 145.03 ( $2 \times \mathrm{NHC}$ aromatic), 145.12 ( $2 \times \mathrm{NHC}$ aromatic), 172,56 ( $2 \times \mathrm{C}=0)$. ESI(+)-MS $\mathrm{CH}_{3} \mathrm{CN}[\mathrm{C}=$ 0.5, SC = 30, EC = 3] m/z (rel. int.): $609\left(100, \mathrm{M}+\mathrm{H}^{+}\right), 462$ (50, M-CONHCHBn + H+ ), 315 (100), 120 (70). HRMS $\mathrm{ES}^{+}$for $\mathrm{C}_{38} \mathrm{H}_{37} \mathrm{~N}_{6} \mathrm{O}_{2} \mathrm{~m} / \mathrm{z}:[\mathrm{M}+\mathrm{H}]^{+}$Calc. 609.2978, found: 609.2975.

2,2'-(1,4-Phenylene)bis-[5-(2-methylthioethyl)-3-(phenylamino)imidazolidin-4-one] (50). Orange solid, $\mathrm{mp}$ $125-127{ }^{\circ} \mathrm{C} . \mathrm{R}_{f} 0.45$ (EtOAc/c- $\mathrm{C}_{6} \mathrm{H}_{12}$ 1:1). IR (neat), $v_{\max }\left(\mathrm{cm}^{-1}\right): 3284,2916,1695,1601,1445,1409,1091 .{ }^{1} \mathrm{H}$ $\operatorname{NMR}\left(300 \mathrm{MHz}, \mathrm{CDCl}_{3}\right): \delta_{\mathrm{H}} 2.00(2 \mathrm{H}, \mathrm{s}, \mathrm{NH}), 2.00-2.04\left(4 \mathrm{H}, \mathrm{m}, \mathrm{CH}_{2}\right), 2.30\left(6 \mathrm{H}, \mathrm{s}, \mathrm{CH}_{3}\right), 2.62-2.64\left(4 \mathrm{H}, \mathrm{m}, \mathrm{CH}_{2}\right)$, 3.74-3.77 (2H, m, CH)), $4.03(2 \mathrm{H}, \mathrm{s}, \mathrm{CH}), 5.92(2 \mathrm{H}, \mathrm{s}, \mathrm{NHPh}), 6.92\left(2 \mathrm{H}, \mathrm{t},{ }^{3} \mathrm{~J}_{H H} 7.9 \mathrm{~Hz}\right), 7.12\left(4 \mathrm{H}, \mathrm{d},{ }^{3} \mathrm{JHH} 8.7 \mathrm{~Hz}\right)$, 7.24-7.27 (4H, m, CH aromatic), $7.40\left(4 \mathrm{H}, \mathrm{dd}, 3^{3} \mathrm{HH}_{3} 8.7,3^{3} \mathrm{JH} 7.9 \mathrm{~Hz}\right) .{ }^{13} \mathrm{C} \mathrm{NMR}\left(75 \mathrm{MHz}, \mathrm{CDCl}_{3}\right): \delta_{\mathrm{C}} 15.40(2 \mathrm{x}$ $\left.\mathrm{SCH}_{3}\right), 30.50\left(\mathrm{CH}_{2}\right), 30.53\left(\mathrm{CH}_{2}\right), 34.10\left(2 \times \mathrm{CH}_{2}\right), 34.14\left(2 \times \mathrm{CH}_{2}\right), 68.00(2 \times \mathrm{CH}), 68.03(2 \times \mathrm{CH}), 75.80(2 \times \mathrm{CH})$, $75.83(2 \times \mathrm{CH}), 113.22(2 \times \mathrm{CH}$ aromatic), $123.11(2 \times \mathrm{CH}$ aromatic), $126.00(\mathrm{CH}$ aromatic), $130.40(\mathrm{CH}$ aromatic), 136.50 ( $2 \times \mathrm{C}$ aromatic), $153.31(2 \times \mathrm{NHC}$ aromatic), $173.20(2 \times \mathrm{C}=0), 173.22$ ( $2 \times \mathrm{C}=0)$. ESI(+)-MS $\mathrm{CH}_{3} \mathrm{CN}[\mathrm{C}=0.5, \mathrm{SC}=30, \mathrm{EC}=3] \mathrm{m} / z$ (rel. int.): $577\left(100, \mathrm{M}+\mathrm{H}^{+}\right), 486(10), 446\left(5, \mathrm{M}-\mathrm{COCH}\left(\mathrm{CH}_{2}\right)_{2} \mathrm{SMeNH}+\mathrm{H}^{+}\right)$, 355 (10). HRMS ES for $\mathrm{C}_{30} \mathrm{H}_{37} \mathrm{~N}_{6} \mathrm{O}_{2} \mathrm{~S}_{2} \mathrm{~m} / \mathrm{z}$ : $[\mathrm{M}+\mathrm{H}]^{+}$Calc. 577.2419, found: 577.2418 .

\section{Acknowledgements}

The authors thank the DGRSRT (Direction Générale de la Recherche Scientifique et de la Rénovation Technologique) of the Tunisian Ministry of Higher Education and Scientific research for financial support.

\section{Supplementary Material}

Stereochemistry analysis, examples of ${ }^{1} \mathrm{H}$ and ${ }^{13} \mathrm{C}$ NMR spectra are available as supporting information.

\section{References}

1. Xu, Z.; Buechler, T.; Wheeler, K.; Wang, H. Chem. Eur. J. 2010, 16, 2972-2976.

https://doi.org/10.1002/chem.200903508

2. Dutta, S. Acta Pharm. 2010, 60, 229-235. https://doi.org/10.2478/v10007-010-0011-1

3. Gupta, P.; Hameed, S.; Jain, R. Eur. J. Med. Chem. 2004, 39, 805-814. https://doi/org/10.1016/i.ejmech.2004.05.005 
4. Antolini, M.; Bozzoli, A.; Ghiron, C.; Kennedy, G.; Rossi, T.; Ursini, A. Bioorg. Med. Chem. Lett. 1999, 9, 1023-1028. https://doi.org/10.1016/S0960-894X(99)00112-2

5. Ellis, G. P.; Epstein, C.; Fitzmaurice, C.; Golberg, L.; Lord, G. H. J. Pharm. Pharmacol. 1964, 16, 400-407. https://doi.org/10.1111/j.2042-7158-1964.tb07482.x

6. Ram, V. J.; Vanden Berghe, D. A.; Vlietinck, A. J. Liebigs. Ann. Chem. 1987, 797-801. https://doi.org/10.1002/jlac.198719870831

7. Tanaka, F.; Takeuchi, S.; Tanaka, N.; Yonehara, H.; Umezawa, H.; Sumiki, Y. J. Antibiot. 1961, 14, $161-162$.

8. Nicolaou, K. C.; Xu, J. Y.; Kim, S.; Pfefferkorn, J.; Ohshima, T.; Vourloumis, D.; Hosokawa, S. J. Am. Chem. Soc. 1998, 34, 8661-8673.

https://doi.org/10.1021/ja981062g

9. Trachsel, A.; Buchs, B.; Godin, G.; Crochet, A.; Fromm, M.; Herrmann, A. Eur. J. Org. Chem. 2012, 28372854.

https://doi.org/10.1002/ejoc.201200081

10. Chambel, P.; Capela, R.; Lopes, F.; Iley, J.; Morais, J.; Gouveia, L.; Gomes, J. R. G.; Gomes, P.; Moreira, R. Tetrahedron 2006, 62, 9883-9891.

https://doi.org/10.1016/j.tet.2006.08.026

11. Illia, P.; Pavel, D.; Zdenka, P.; Jiri, H.; Milos, S. J. Heterocyclic Chem. 2010, 47, 1356-1360. https://doi.org/10.1002/jhet.454

12. Illia, P.; Pavel, D.; Zdenka, P.; Petr, S.; Milos, S. J. Org. Chem. 2011, 76, 4787-4793. https://doi.org/10.1021/jo200703j

13. Pavel, D.; Sergej, K.; Illia, P.; Milos, S. Tetrahedron: Asymmetry 2013, 24, 334-339. https://doi.org/10.1016/j.tetasy.2013.02.007

14. Bahy, A.; Kacem, Y.; Ben Hassine, B. Synthetic Commun. 2010, 40, 1377-1390. https://doi.org/10.1080/00397910903097237

15. Kacem, Y.; Kraiem, J.; Kerkani, E.; Bouraoui, A.; Ben Hassine, B. Eur. J. Pharm. Sci. 2002, 16, 221-228. https://doi.org/10.1016/S0928-0987(02)00046-5

16. Aliyenne, A. O.; Khiari, J. E.; Kraïem, J.; Kacem, K.; Ben Hassine, B. Tetrahedron Lett. 2006, 47, 6405-6408. https://doi.org/10.1016/i.tetlet.2006.06.148

17. Aliyenne, A. O.; Kraïem, J.; Kacem, J.; Ben Hassine, B. Tetrahedron Lett. 2008, 49, 1473-1475. https://doi.org/10.1016/j.tetlet.2008.01.015

18. Tka, N.; Kraiiem, J.; Kacem, Y.; Hajri, A.; Ben Hassine, B. C. R. Chimie 2009, 12, 1066-1071. https://doi.org/10.1016/i.crci.2008.09.004

19. Kacem, Y.; Ben Hassine, B. Tetrahedron Lett. 2012, 53, 5608-5610. https://doi.org/10.1016/i.tetlet.2012.08.008

20. Kacem, Y.; Ben Hassine, B. Tetrahedron: Asymmetry 2014, 25, 252-257. https://doi.org/10.1016/j.tetasy.2013.12.002

21. Kacem, Y.; Ben Hassine, B. Heterocycles 2014, 89, 197-207. https://doi.org/10.3987/COM-13-12878

22. Verardo, G.; Toniutti, N.; Gorassini, A.; Giumanini, A.G. Eur. J. Org. Chem. 1999, 2943-2948. https://doi.org/10.1002/(SICI)1099-0690(199911)1999:11<2943::AID-EJOC2943>3.0.CO;2-X 